\title{
Lens design with automatic differentiation
}

\author{
Congli Wang, Ni Chen, and Wolfgang Heidrich \\ Visual Computing Center, King Abdullah University of Science and Technology, Thuwal 23955, Saudi Arabia \\ \{congli.wang, ni.chen, wolfgang.heidrich\}@kaust.edu.sa
}

\begin{abstract}
We propose to power lens design ray-tracing engines with automatic differentiation. Along with the primal, derivatives are algorithmically obtained for lens parameters. Exploiting the gradient information, applications are shown including design optimization and sensitivity analysis. (C) 2021 The Author(s)
\end{abstract}

\section{Lens design with automatic differentiation}

Inspired by the recent success in machine learning and differentiable rendering, we propose to power lens design with automatic differentiation. As in Fig. 1, a lens system is described as a concatenation of multiple surfaces that are parameterized by $\boldsymbol{\theta} \in \mathbb{R}^{n}$, e.g., surface curvature, asphere coefficients, spacing to next surface, and etc. Starting from an initial ray of known origin $\mathbf{o} \in \mathbb{R}^{3}$, direction $\mathbf{d} \in \mathbb{R}^{3}$, and wavelength $\lambda \in \mathbb{R}$, a computational engine performs sequential ray-tracing through the surfaces, intersecting the sensor plane at transversal point $\mathbf{p} \in \mathbb{R}^{2}$, following geometric optics and Snell's law. This design-specific ray-tracing process can be formulated as $\mathbf{p}(\mathbf{o}, \mathbf{d}, \lambda ; \boldsymbol{\theta})$. The computation is black-box, nonlinear, and can only be numerically evaluated. This numerical evaluation can be implemented along with automatic differentiation [1], where not only $\mathbf{p}$ (the primal) but also $\partial \mathbf{p} / \partial \boldsymbol{\theta} \in \mathbb{R}^{n \times 2}$ (the derivatives, here the transposed Jacobian) are obtained through the computation, as in Fig. 1.

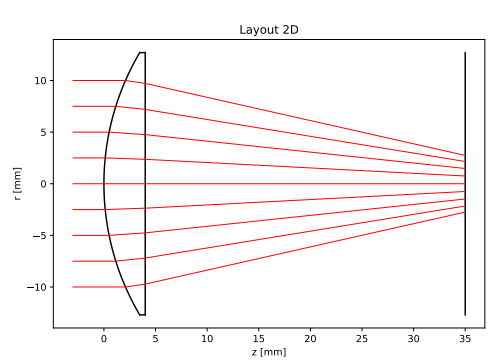

(a) Singlet of curvatures $c_{1}, c_{2}$

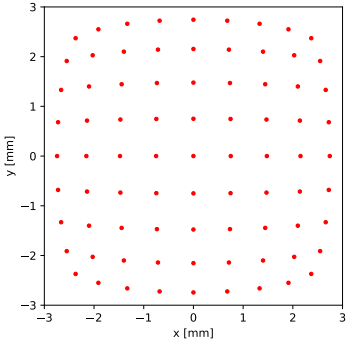

(b) $\mathbf{p}\left(c_{1}, c_{2}\right)$

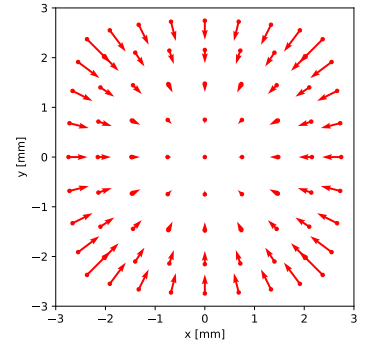

(c) $\partial \mathbf{p} / c_{1}$

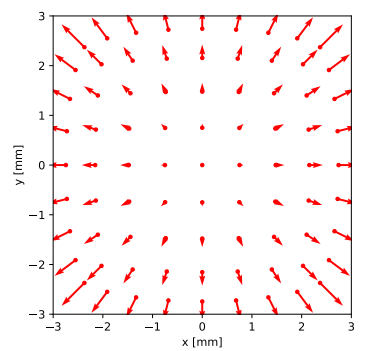

(d) $\partial \mathbf{p} / c_{2}$

Fig. 1: A lens system parameterized by surface parameters $\boldsymbol{\theta}=\left(c_{1}, c_{2}\right)$, the two surface curvatures here. With automatic differentiation, spot diagram contains both the primal $\mathbf{p}$ and its derivatives $\partial \mathbf{p} / \partial \boldsymbol{\theta}=\left(\partial \mathbf{p} / \partial c_{1}, \partial \mathbf{p} / \partial c_{2}\right)$.

\section{Applications}

To optimize or tolerancing a lens design, additional analysis needs to be performed on intersection points $\mathbf{p}$, for example in spot diagram analysis a root-mean-square (RMS) spot error is obtained by tracing rays sampled within the entrance pupil at specific viewing angles. This deterministic analysis, denoted as scalar function $f(\cdot)$, takes sampled $\mathbf{p}_{i}(\boldsymbol{\theta})=\mathbf{p}\left(\mathbf{o}_{i}, \mathbf{d}_{i}, \lambda_{i} ; \boldsymbol{\theta}\right)$ as inputs, and outputs an error metric $\varepsilon(\boldsymbol{\theta}) \in \mathbb{R}$, also known as the merit function:

$$
\varepsilon(\boldsymbol{\theta})=\sum_{i} f\left(\mathbf{p}_{i}(\boldsymbol{\theta})\right), \quad \text { and its derivatives } \quad \frac{\partial \varepsilon}{\partial \boldsymbol{\theta}}=\sum_{i} \frac{\partial \mathbf{p}_{i}}{\partial \boldsymbol{\theta}} \frac{\partial f}{\partial \mathbf{p}_{i}} .
$$

\subsection{Design optimization}

In design optimization, the goal is to find a set of optimal parameters $\boldsymbol{\theta}^{*}$ that minimizes $\varepsilon(\boldsymbol{\theta})$ in Eq. (1). Once $\partial \varepsilon / \partial \boldsymbol{\theta}$ is available, gradient descent can be employed for this purpose, given a learning rate $\alpha$ :

$$
\boldsymbol{\theta}^{*}=\arg \min _{\boldsymbol{\theta}} \varepsilon(\boldsymbol{\theta}), \quad \text { solved by } \quad \boldsymbol{\theta}^{(k+1)} \leftarrow \boldsymbol{\theta}^{(k)}-\left.\alpha \frac{\partial \varepsilon}{\partial \boldsymbol{\theta}}\right|_{\boldsymbol{\theta}=\boldsymbol{\theta}^{(k)}} .
$$

In Fig. 2, a pre-computer age design [2] is being optimized using the proposed technique, with $\boldsymbol{\theta}$ being all the surface curvatures. Here, $\boldsymbol{\varepsilon}(\boldsymbol{\theta})$ is the summation of all the RMS spot errors at three different viewing angles, at $\lambda=587.6 \mu \mathrm{m}$. The optimized design performs better compared to the original initial design in terms of $\varepsilon(\boldsymbol{\theta})$. 

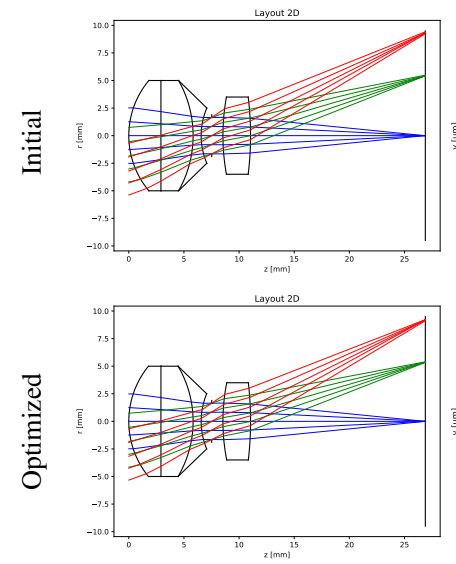

(a) Lens system
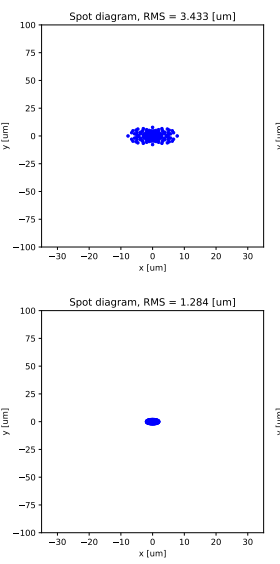

(b) $\mathbf{p}$ spot diagrams at three viewing angles $0^{\circ}, 12^{\circ}$, and $20^{\circ}$

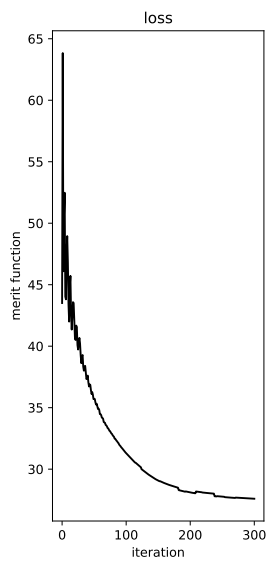

(c) $\varepsilon(\boldsymbol{\theta})$ versus $k$

Fig. 2: Design optimization by gradient descending $\boldsymbol{\theta}$.

\subsection{Sensitivity analysis}

In tolerancing a lens system, or sensitivity analysis, a presuming small, linear parameter perturbation $\Delta \boldsymbol{\theta}$ is enforced, and the total effect of the perturbation, $\Delta \varepsilon$, is calculated through the nominal system $\boldsymbol{\theta}_{0}$ to determine the potential effects. This is mathematically paraphrased by relating $\Delta \varepsilon$ to $\Delta \boldsymbol{\theta}$ through the derivatives in Eq. (1):

$$
\Delta \boldsymbol{\varepsilon}=\left.\Delta \boldsymbol{\theta} \cdot \frac{\partial \varepsilon}{\partial \boldsymbol{\theta}}\right|_{\boldsymbol{\theta}=\boldsymbol{\theta}_{0}}, \quad \text { or in the vector form } \quad \Delta \boldsymbol{\varepsilon}=\left[\Delta \varepsilon_{1}, \Delta \varepsilon_{2}, \cdots, \Delta \varepsilon_{m}\right]^{T}=\frac{\partial \boldsymbol{\varepsilon}}{\partial \boldsymbol{\theta}} \Delta \boldsymbol{\theta} .
$$

In sensitivity analysis, $\Delta \boldsymbol{\theta}$ is given to compute $\Delta \boldsymbol{\varepsilon}$, whereas in inverse sensitivity analysis, $\Delta \boldsymbol{\varepsilon}$ is given to compute $\Delta \boldsymbol{\theta}$, assuming equal contribution from the error sources, or weighted according to their prior probabilities. In Fig. 3, an optimized version of the original double-Gauss [3] is under sensitivity analysis, with all surface curvatures $\boldsymbol{\theta}_{c}$ (excluding the aperture one) and surface spacing $\boldsymbol{\theta}_{d}$ being toleranced. The proposed automatic differentiation engine can obtain the Jacobian matrix $\partial \boldsymbol{\varepsilon} / \partial \boldsymbol{\theta}$ known as the sensitivity matrix for further analysis.
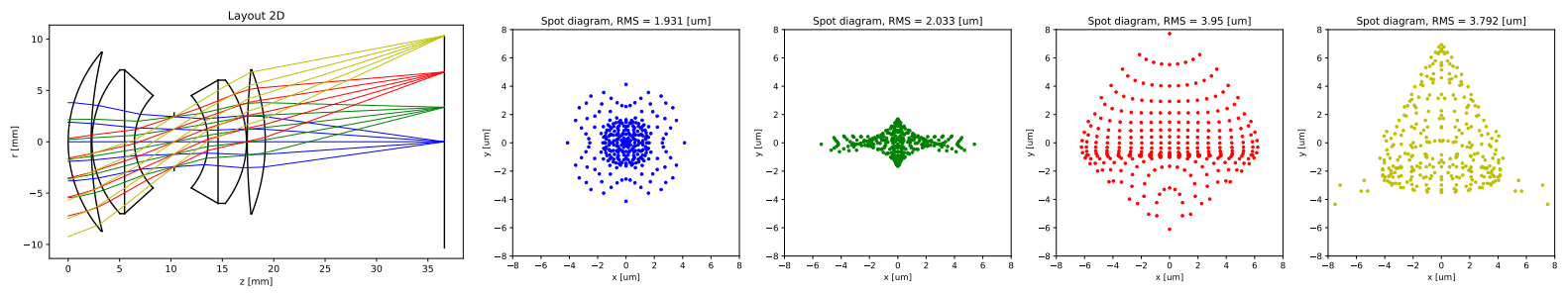

(a) Lens tolerancing $\boldsymbol{\theta}=\left(\boldsymbol{\theta}_{c}, \boldsymbol{\theta}_{d}\right)$
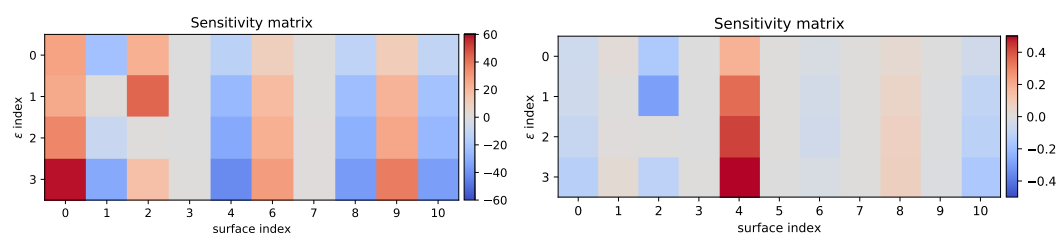

(c) Sensitivity matrices $\partial \boldsymbol{\varepsilon} / \partial \boldsymbol{\theta}_{c}$ and $\partial \boldsymbol{\varepsilon} / \partial \boldsymbol{\theta}_{d}$

Fig. 3: Sensitivity analysis for an existing design.

\section{References}

1. A. G. Baydin, B. A. Pearlmutter, A. A. Radul, and J. M. Siskind, "Automatic differentiation in machine learning: a survey," J. Mach. Learn. Res. 18 (2018).

2. H. Sylvester, "Photographic objective," (1938). U.S. Patent 2124301A.

3. J. G. Baker, "Highly corrected objective having two inner divergent meniscus components between collective components," (1949). U.S. Patent 2532751. 